\title{
Large-area flexible nanostripe electrodes featuring plasmon hybridization engineering
}

\author{
Carlo Mennucci ${ }^{\S}$, Debasree Chowdhury ${ }^{\S}$, Giacomo Manzato, Matteo Barelli, Roberto Chittofrati, Christian Martella ${ }^{\dagger}$, \\ and Francesco Buatier de Mongeot $(\Delta)$
}

Dipartimento di Fisica, Università degli Studi di Genova, via Dodecaneso 33, I-16146, Genova, Italy

${ }^{\dagger}$ Present address: CNR-IMM Unit of Agrate Brianza, via C. Olivetti 2, Agrate Brianza, I-20864, Italy

${ }^{\S}$ Carlo Mennucci and Debasree Chowdhury contributed equally to this work.

(C) The Author(s) 2020

Received: 28 February 2020 / Revised: 11 September 2020 / Accepted: 14 September 2020

\begin{abstract}
Multifunctional flexible Au electrodes based on one-dimensional (1D) arrays of plasmonic gratings are nanofabricated over large areas with an engineered variant of laser interference lithography optimized for low-cost transparent templates. Au nanostripe (NS) arrays achieve sheet resistance in the order of $20 \mathrm{Ohm} / \mathrm{square}$ on large areas $\left(\sim \mathrm{cm}^{2}\right)$ and are characterized by a strong and dichroic plasmonic response which can be easily tuned across the visible (VIS) to near-infrared (NIR) spectral range by tailoring their cross-sectional morphology. Stacking vertically a second nanostripe, separated by a nanometer scale dielectric gap, we form near-field coupled $\mathrm{Au} / \mathrm{SiO}_{2} / \mathrm{Au}$ dimers which feature hybridization of their localized plasmon resonances, strong local field-enhancements and a redshift of the resonance towards the NIR range. The possibility to combine excellent transport properties and optical transparency on the same plasmonic metasurface template is appealing in applications where low-energy photon management is mandatory like e.g., in plasmon enhanced spectroscopies or in photon harvesting for ultrathin photovoltaic devices. The remarkable lateral order of the plasmonic NS gratings provides an additional degree of freedom for tailoring the optical response of the multifunctional electrodes via the excitation of surface lattice resonances, a Fano-like coupling between the broad localised plasmonic resonances and the collective sharp Rayleigh modes.
\end{abstract}

\section{KEYWORDS}

nanofabrication, nanostripes, transparent electrodes, plasmonic dimers, interference lithography, surface lattice resonances

\section{Introduction}

Plasmonics has gained considerable momentum due to the ever increasing ease in the methods of fabrication and characterization of optical nanoantennas and also thanks to their multidisciplinary applications [1-5]. Indeed, localized surface plasmon (LSP) resonance, the collective oscillation of conduction electrons in noble metal nanoparticles resonantly driven by external electromagnetic fields, represents one of the most powerful ways to manipulate light at the nanoscale. Functional devices incorporating plasmonic nanoparticles on rigid dielectric or semiconducting templates have been employed in proof of concept seminal experiments [6-10]. More recently, the research interest is turning towards the development of advanced multifunctional plasmonic architectures, supported onto flexible, nonplanar and transparent polymer substrates [11-14] with tailored LSP resonances for their promising potential in biosensing [15, 16], refractive-index sensing [17], wire-grid polarizer [18-20] and opto-electronics [21, 22], etc. A crucial advancement is represented by the possibility to exploit, at the same time, the plasmonic properties of noble metal nanowire arrays together with their excellent electrical transport properties to be employed e.g., as multifunctional transparent conductive electrodes (TCE) for flexible devices with added functionality of plasmon-enhanced photon harvesting [23-27].
Conventional transparent conductive electrodes based on oxides such as indium tin oxide (ITO) suffer from serious delamination issues when applied to flexible devices such as electronic displays [28], touch screens, smart windows, flexible electronics [29] or transparent heaters [30] and a growing demand of alternative transparent electrodes based on metal nanowire networks is observed.

The possibility to shift the LSP resonance of the metal nanostructure from the visible towards the near-infrared (NIR) spectral range represents an important issue for increasing energy harvesting efficiency in photodetection and photovoltaic applications. Also, in plasmon-enhanced nanospectroscopy applications, like e.g., surface-enhanced Raman spectroscopy (SERS), a shift of the resonant LSP and pump laser frequency towards the NIR is mandatory for avoiding light induced photochemical degeneration and for achieving efficient detection of the weak Raman signal of biological molecules due to the reduction of the fluorescence background $[31,32]$. In a more conventional way, the LSP resonance can be tuned towards the NIR by modifying the geometrical parameters of the individual metal nanostructures (shape, size, and surrounding medium) [33-36]. Alternatively, more complex architectures allow to redshift the LSP resonance exploiting plasmon hybridization in ensembles of closely coupled dimeric or multimeric nanostructures [37-39]. Indeed, similarly to what happens with 
atomic orbitals in a molecule, when two resonating plasmonic particles are placed in close proximity, their local EM near fields interact and give rise to two hybrid resonances, one with a bonding and another with an anti-bonding character, located at different energies [40]. These are interpreted as magnetic dipole (MD) and electric dipole (ED) resonances which respectively correspond to the out-of-phase and in-phase oscillation of dipoles. Particular attention is devoted to the MD mode, also known as gap-plasmon resonance, due to the possibility to redshift the dipolar resonant frequency towards the NIR range. A recent work from some of the authors [36] has demonstrated that large-area one-dimensional (1D) arrays of $\mathrm{Au} / \mathrm{SiO}_{2} / \mathrm{Au}$ stacked nanostripe dimers, featuring LSP hybridization, can be synthesized by grazing incidence deposition on self-organized (SO) rippled glass templates. In that case however, due to the intrinsic size dispersion of the SO pattern, a spectrally broad MD resonance was observed. The lack in spectral selectivity of the MD resonance is detrimental when it is mandatory to match the narrow spectral lines (e.g., of a molecular transition or of a laser pump) for maximizing the efficacy of plasmonic near-field nanospectroscopy applications. All above cited examples, in which plasmon hybridization takes place by near-field coupling of isolated and electrically nonconductive nanoclusters, highlight the need of a compromise between large area and high resolution nanofabrication in order to achieve multifunctional templates endowed with optical transparency, electrical conductivity and tunable plasmon hybridization via near-field coupling.

In this paper we address these issues using a novel variant of laser interference lithography (LIL) which enables the production of plasmonic structures periodically arranged over large area $\left(\mathrm{cm}^{2}\right.$ scale), on low cost, transparent and flexible substrates (e.g., soda-lime glasses or polymer films like Arylite ${ }^{\mathrm{TM}}$ ). Among all possible geometries we explored the $1 \mathrm{D}$ case since the nanostripes (NSs) can sustain LSP resonances when light is polarized transversely and meanwhile can also work as anisotropic transparent electrodes when the contacts are placed in the longitudinal direction. In particular, we have grown $\mathrm{Au}$ NSs directly on glass or polymer substrates, typical transparent window layers employed in photovoltaic or bio-sensing applications. Due to the high quality of the LIL nanofabrication process, the Au NSs array show low sheet resistances in the $10-20 \mathrm{Ohm} / \mathrm{sq}$ range; this feature, conjugated with high optical dichroism and high transmittance in the Vis-NIR, makes them competitive with ITO-based transparent conductive oxides commonly used in optoelectronics [41].

We also demonstrate that the LIL nanofabrication process can be extended in the third dimension to produce dimeric plasmonic structures consisting of vertically stacked $\left(\mathrm{Au} / \mathrm{SiO}_{2} / \mathrm{Au}\right)$ nanostripes separated by $\mathrm{nm}$-scale dielectric gap. Near-field hybridization of the LSP resonances of the individual nanostripes constituting the dimers leads to the appearance of sharp and spectrally resolved MD mode, which is redshifted into the NIR with respect to the plasmon mode of the isolated NS constituents. Additionally, thanks to the highly periodic lateral arrangement of the plasmonic nanostripes, it is possible to engineer coherent light scattering from the array into sharp optical modes (Rayleigh anomalies) peaked at wavelengths related with the pattern periodicity. The spectral overlap of such dispersive propagating modes with the LSP resonances of the NS monomers or dimers strongly amplifies the local electromagnetic field [42] and qualifies the transparent electrodes also as multifunctional templates featuring enhanced photon harvesting in the NIR spectral range with possible applications ranging from nanospectroscopy and biosensing to photovoltaics. In these experiments we employed $\mathrm{Au}$ as the active plasmonic element due to its chemical stability towards oxidation and tarnishing, as well as, to its excellent electrical conductivity. We stress however that the described nanofabrication method could be adapted easily to other elements relevant for plasmonics thus providing a further handle for tailoring the plasmonic response.

\section{Results and discussion}

Top-down nanofabrication approaches (e.g., EBL) allow complex design of nano-resonators, but are extremely time consuming and costly if patterned regions have to be in the $\mathrm{cm}^{2}$ scale. To solve this issue, parallel nanopatterning techniques have been developed, such as nanoimprint or nanostencil lithography. The latter approaches however rely on the use of an expensive master, fabricated via cumbersome serial processes as focused ion beam, EBL or high resolution lithography. Further drawbacks are related with master or mask deterioration and to the limited versatility in the design of the mask. Conversely, large area and low cost nanofabrication can be achieved via bottom-up approaches based on self-assembly or self-organization, e.g., recurring to defocused ion beam sputtering (IBS) [43-45] at the expense of a reduced uniformity of the patterns on large scale.

Here, we develop a low-cost variant of an interference lithography setup modified for the fabrication of conductive plasmonic nanoarrays extending uniformly over transparent and flexible substrates on large areas at the $\mathrm{cm}^{2}$ level. Typically, when LIL is performed on high refractive index substrates such as silicon or metals, an anti-reflection coating is applied in order to prevent strong reflections of light from the front interface towards the resist layer. In the present case we instead focus on low refractive index transparent substrates (glasses or a commercial polycarbonate polymer such as Arylite ${ }^{\mathrm{TM}}$ ) for which front reflectance is weak, while transmission and back reflectance are significant and can lead to the formation of interference fringes which in turn perturb the desired pattern in the photoresist. The LIL system sketched in Fig. 1 consists of an ultra-compact and cheap semiconductor laser diode emitting at $406 \mathrm{~nm}$ (Ondax LM406-PLR40) followed by a spatial filter consisting in an aspheric biconvex lens, a $20 \mu \mathrm{m}$ pinhole, a metallic holder for mirror (Edmund Optics, surface roughness $\lambda / 10$, Enhanced Aluminium) and sample. Mirror and sample are mounted at a relative angle of $90^{\circ}$ from each other, on a rotating stage at a distance $(D)$ of $1.5 \mathrm{~m}$ from the pinhole in a Lloyd's mirror Interference configuration [46].

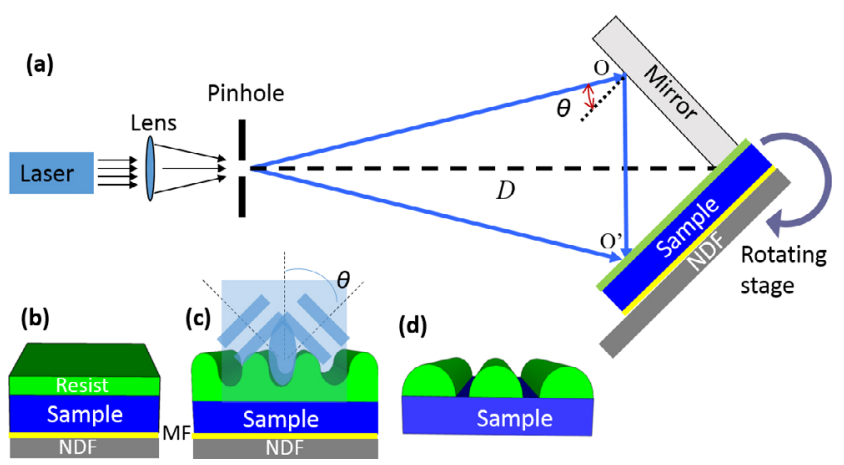

Figure 1 Sketch of LIL setup used for large area nanopatterning of polymeric thin film (thin green slab) spin coated on the sample (glass or polymer). (b)-(d) are the consecutive steps to be taken to complete the patterning process: firstly, a thin photoresist film is spun on the substrate coupled to a neutral density filter through matching fluid (b), afterwards interference fringes are recorded into the resist layer (c) and thanks to wet development of the resist layer a final rippled morphology is obtained (d). 
If the distance $D$ is large enough, the incident waves at the mirror/sample position can be considered as nearly planar, thus the symmetric points $\mathrm{O}$ and $\mathrm{O}^{\prime}$ are illuminated by an in-phase wave front (see Fig. 1(a)). A fraction of the incident beam is reflected by the mirror toward the sample where, due to the difference in the optical path, it interferes with the direct light beam and generates a sinusoidal standing wave which impresses the photoresist film spincoated on the sample.

The period of the pattern $P$, expressed in terms of the laser wavelength $\Lambda$ and of the angle $\theta$ formed by the mirror and the laser axis through the relation $P=\lambda /(2 \sin \theta)$, can be tuned in a broad range by simply modifying the incidence angle $\theta$ as sketched in Fig. 1(a). In order to address the issue of reflection at the back interface of the transparent substrate and the subsequent formation of interference fringes in the photoresist we devised an original solution which consists in coupling the transparent substrate to a neutral density filter (NDF) with high optical density (OD: 4.00) by placing in between a refractive index matching fluid (MF) (Cargille immersion liquid, OHGL $\mathrm{nD}$ ) (sketched in Figs. 1(b)-1(d)). In this way internal reflections are minimised and the laser light transmitted through the sample can be totally absorbed inside the ND filter. Further details of the LIL lithography steps are discussed in experimental section.

The so devised LIL process enables the fabrication of highly ordered periodic arrays of grooves on a flexible substrate (Arylite ${ }^{\mathrm{TM}}$ ) as revealed by the photograph shown in Fig. 2(a). The patterns, here realised with a laser angle of incidence $\theta=45^{\circ}$, extend uniformly over $\mathrm{cm}^{2}$ area as revealed by coherent scattering of light from the surface grating. Comparable patterns are formed on low-cost soda lime glass substrates following the same LIL protocol. The atomic force microscopy (AFM) image of Fig. 2(b), which refers to the latter case, demonstrates the high degree of nanoscale order of the LIL pattern. In order to quantitatively estimate the periodicity of the pattern we used the $2 \mathrm{D}$ self-correlation function of the AFM image. A zoom of the generated self-correlation function $G(r)$ is shown in the inset of Fig. 2(b) and its corresponding line profile, orthogonal to the ridges, is plotted in the black trace of Fig. 2(c). The period $P$, estimated by measuring the distance between two consecutive maxima in the $G(r)$ line profile, reads $293 \pm 6 \mathrm{~nm}$, in agreement with the theoretical one $(P=\lambda / 2 \sin \theta)$ within the experimental error of about $1^{\circ}$ in the mirror rotation stage. LIL experiments have been also realised for laser light incidence angles $\theta=40^{\circ}$ and $35^{\circ}$. In each case an approximately sinusoidal pattern formation is achieved as demonstrated by AFM analysis (Fig. 2(d)) and the measured periodicity, extracted from $G(r)$ in Fig. 2(c), respectively reads $328 \pm 5$ and $352 \pm 6 \mathrm{~nm}$. A coarser tuning of the pattern periodicity $P$ from 250 up to $1,200 \mathrm{~nm}$ has been also achieved by simply varying the laser incidence angle $\theta$ from $55^{\circ}$ to $10^{\circ}$ respectively (see AFM micrographs in Fig. S1 in the Electronic Supplementary Material (ESM)). A numerical analysis of the AFM topography $z(x, y)$ shown in Fig. 2(b) also allows to calculate the firstderivative function $\mathrm{d} z / \mathrm{d} x$ along the fast scan direction $x$ which is symmetrically peaked at $\phi \sim \pm 59^{\circ}$ for LIL incidence angle $\theta$ set at $45^{\circ}$ and this value is found shifted to $\pm 57^{\circ}$ and $\pm 55^{\circ}$ for $\theta=40^{\circ}$ and $35^{\circ}$ respectively. The possibility to fabricate large area dielectric templates with periodically arranged steep facets represents a natural platform for the confinement of plasmonic nanostripe arrays employing glancing angle deposition of noble metals according to the sketch of Fig. 2(d). A similar approach was previously followed in the case of self-organised faceted glass templates prepared by ion beam sputtering $[9,47$, 48] which highlighted the possibility to fabricate plasmonic arrays with a broadband response determined by inhomogeneous broadening i.e., by the incoherent superposition of the plasmonic response of $\mathrm{Au}$ nanoparticles with an intrinsically broad size (a)

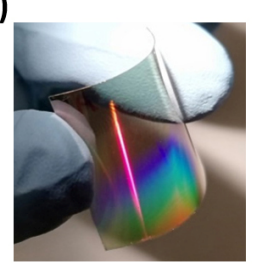

(b)

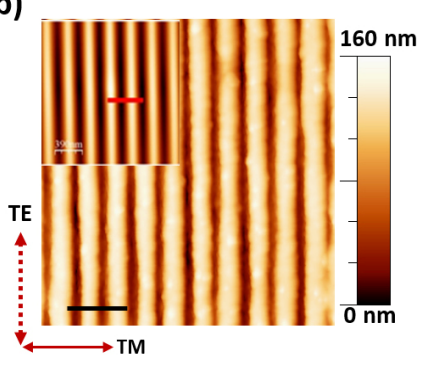

(c)

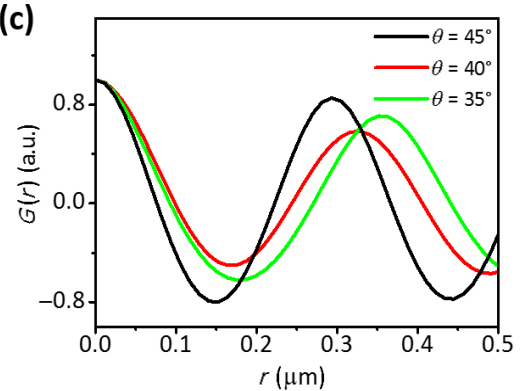

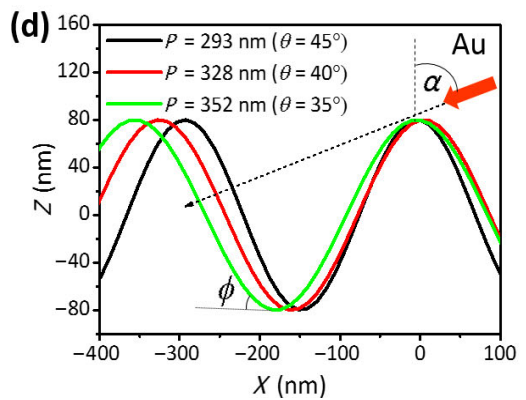

(e)

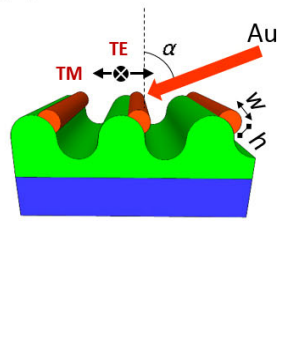

Figure 2 (a) A photograph shows large area NSs array grown on flexible (Arylite ${ }^{\mathrm{TM}}$ ) template when the laser angle of incidence is set at $\theta=45^{\circ}$. (b) An AFM topography acquired on LIL nanopatterned photoresist (AZ MIR 701) shown in (a). In the inset, the 2D auto correlation function G( $r$ ) of the AFM image is reported where the scale bar corresponds $630 \mathrm{~nm}$. (c) Black trace is the line profile extracted from $G(r)$ function represented in (b) while red and green traces corresponds to $G(r)$ profiles extracted from AFM images acquired on LIL patterns realized at different laser interference conditions. (d) AFM line profiles show the increase of period when the laser incidence angle $\theta$ is modified from $45^{\circ}$, to $40^{\circ}$ and $35^{\circ}$. The projection of the Au atomic beam at grazing angles $\alpha$ highlights the shadowing mechanism exploited to synthesize tilted Au NSs. $\phi$ indicates the slope of the nanopattern facet. (e) A sketch of the grazing incidence deposition of Au NSs on rippled polymer. (f) Far-field optical transmission measurements in extinction configuration on Au nanowires confined by grazing incidence metal evaporation on sinusoidal resist patterns with three different periodicities. The arrows indicate transmission minimum. The optical spectra are measured with incident light polarized orthogonally (TM-solid line) and parallel (TE-dashed line) to nanowire axis as highlighted in the sketch in (b) and (e). 
dispersion inherent to the self-organised process.

\subsection{Tilted nanostripes}

Here, we exploit the highly ordered undulated resist pattern realized by LIL both on low cost glasses and on flexible polymer substrates (Arylite ${ }^{\mathrm{TM}}$ ) in view of template-driven metal nanoparticle agglomeration exploiting shadowing mechanism in order to reduce the degree of lateral disorder of individual $\mathrm{Au}$ nanoparticles and the dispersion in their size distribution. More in detail, we thermally evaporated an Au film at a tilted angle $\alpha=70^{\circ}$ off-normal (the equivalent thickness deposited on the flat substrate is $t=5.3 \mathrm{~nm}$ ) on the undulated resist pattern obtained due to the laser exposure at an angle of $45^{\circ}$. Due to the slope $\left(\phi \sim 59^{\circ}\right)$ of the facets, the local thickness is derived following the equation $h=(t \times \cos (\alpha-\phi)) / \cos \alpha$ $\sim 15.3 \mathrm{~nm}$, with $t=5.3 \mathrm{~nm}, \alpha=70^{\circ}$ and $\phi=59^{\circ}$ respectively (a sketch displaying the nanostripes dimensions and the deposition scheme is presented in panel 2(e) and in Fig. S2 in the ESM). The optical transmission spectra corresponding to TE and TM polarization, i.e., parallel or perpendicular to the NS main axis, are plotted in Fig. 2(f) (black dashed and solid curve respectively) and reveal the strong optical dichroism induced by the arrays of tilted Au NSs. The TE spectrum resembles typical transmittance spectra of a flat gold film with $\mathrm{Au}$ interband transition at $\lambda=520 \mathrm{~nm}$ followed by a monotonic drop in the transmittance moving toward the infrared region, as expected within simple Drude model [49]. On the contrary, in the TM spectra we find the spectral signature of LSP resonance excitation with a broad extinction band centred at $700 \mathrm{~nm}$ and an increased transmittance toward the infrared region. Figure 2(f) also includes the TM spectra of the metal nanostructures, of comparable thickness $h$, deposited onto the sinusoidal pattern obtained at $\theta=40^{\circ}$ and $35^{\circ}$ (red and green traces respectively) under similar LIL experimental conditions to the case of $\theta=45^{\circ}$. While for TE polarization no relevant variations are observed, for TM configuration a clear red-shift of the LSP resonance band from $\lambda_{\text {res }}=680 \mathrm{~nm}$ (black trace) to $\lambda_{\text {res }}=780 \mathrm{~nm}$ (green trace) is found when the sample periodicity increases from 293 to $352 \mathrm{~nm}$. This is mainly due to the morphological modification of the height/width $(h / w)$ aspect ratio of the individual nanostripes. Indeed, the change in the periodicity of the undulated pattern influences the shadowing mechanism for the same Au grazing incidence evaporation condition as better clarified in Fig. 2(d). When evaporation is performed on a template endowed with larger periodicity, a wider portion of the facet side is illuminated by the Au beam leading to an increase of the NS width $w$. In this scenario, Au nanostripes with tunable aspect ratio are achieved by changing their width. Moreover, the decrease of slope $\phi$ of the faceted template with increase of pattern periodicity induces a slight decrease in deposited Au thickness since the local deposition flux scales as $\cos (\alpha-\phi)$. Both the effects contribute to a decrease of the NSs aspect ratio $h / w$ which is at the basis of the observed redshift of plasmonic resonance reported in Fig. 2(f). Summing up, on one side the glancing angle deposition approach ensures a simple one-step approach for the synthesis of tilted NS arrays endowed with tunable plasmonic response, while on the other hand the rounded shape of the sinusoidal template leads to a substantial broadening of the LSP resonance. Another factor which contributes to a broadening of the LSP resonance is related to the tilted orientation of the NSs with respect to the electric field (confined in the horizontal plane) which enables the excitation of the LSP resonance polarized also along the short-axis $(h)$. Finally, we must consider that this approach does not allow to independently control the NS morphological parameters: a modification of the template periodicity $P$ leads to a combined modification of the NS tilt and width which in turn affect its plasmonic response.

\subsection{Flat nanostripes}

In order to solve the above mentioned issues we thus engineered the LIL fabrication in order to use normal incidence deposition conditions of flat metal nanostripes through lithographic masks with independent control on width and periodicity. The AFM topography of Fig. 3(a) represents the stencil mask formed by polymer resist nanostripes with a periodicity $P=$ $300 \mathrm{~nm}$ after laser illumination at an angle $\theta=45^{\circ}$. In order to carefully tune the gap width between polymer lines, we deposited in-situ a double sided metallic overhanging structure formed by $\mathrm{Au}$ evaporated at grazing incidence ( $80^{\circ}$ off-normal) orthogonally to the ripple long axis (see the sketch in Fig. 3(b)). As highlighted in Figs. 3(a) and 3(b), the topographic width of the gap formed by the disconnected polymer lines (green trace) (a)

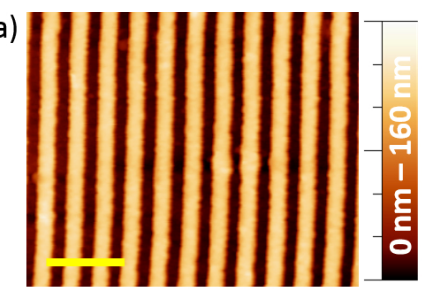

(b)

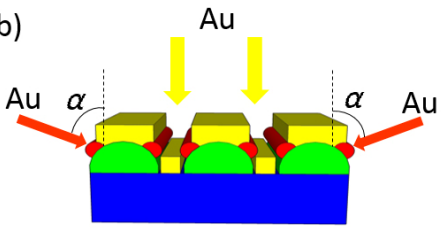

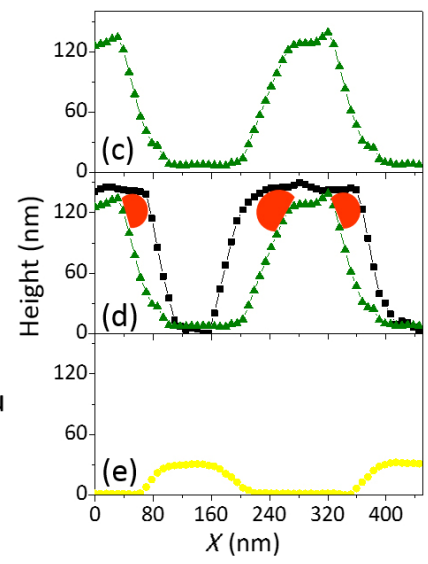

(f)
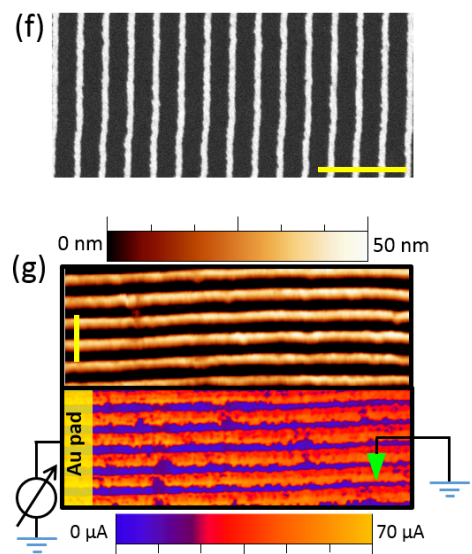

Figure 3 (a) AFM topography acquired on laterally separated polymer resist lines synthesized by LIL at $45^{\circ}$ (Scale bar represents $800 \mu \mathrm{m}$ ). (b) Sketch representing the synthesis of metal overhanging structures evaporated at grazing incidence on both facets of the resist lines and the final evaporation of flat NSs through the opening slit. In (c) topographic line acquired on the resist lines visible in the AFM image (a). In (d) a topographic line (black) acquired on the sample prepared with double sided metal NSs mask is superimposed to the profile of pristine ripple pattern (green). In (e) a topographic profile measured on flat Au NSs evaporated at normal incidence through the NSs mask is reported after lift-off. In (f) a SEM micrograph acquired in the backscattered channel shows Au NSs (width $=96 \mathrm{~nm}$, thickness $=25 \mathrm{~nm}$, period $=300 \mathrm{~nm}$ ). Scale bar represents $1 \mu \mathrm{m}$. In (g) the topography (upper map, yellow line indicates scale bar $650 \mathrm{~nm}$ ) of $35 \mathrm{~nm}$-thick Au NSs has been measured simultaneously to the local electric current (lower map). A virtually grounded AFM tip is scanned in contact with the biased surface, as schemed in the image. 
is modified once the polymer NS is topped by the metal overhangs-black trace in Fig. 3(d). Finally, without breaking vacuum, a $2 \mathrm{~nm}$-thick Ti adhesion layer has been evaporated in-situ at normal incidence immediately before deposition of a $25 \mathrm{~nm}$ thick Au layer. After deposition, the lift-off procedure allowed complete removal of the resist leaving on the substrate an ordered array of flat $\mathrm{Au}$ NSs, whose representative topographic profile is reported in Fig. 3(e). We stress that besides allowing a fine tuning of the opening gap between polymer lines, the overhanging structures also improve lift-off of the polymer mask since shadowing avoids capping of the polymer sidewalls under normal incidence metal deposition. The SEM micrograph reported in Fig. 3(f) demonstrates the high quality of the NS fabrication process, while further data acquired at a lower magnification reveal that the NSs extend uninterrupted for distances exceeding several $\mathrm{mm}$ (see Fig. S3 in the ESM). These morphological observations suggest that such hybrid templates, composed by metal NS supported on polymer/glass templates, can be exploited as multifunctional transparent electrodes endowed with an excellent sheet resistance. Moreover, as the metal NSs are more resilient to bending-induced delamination with respect to common transparent conductive oxides (like e.g., indium doped tin oxide), all the aforementioned plasmonic functionalities can be integrated into flexible transparent electrodes of interest for flexible opto- electronic applications [50].

In Fig. 3(g), we highlight a test of the electrical transport properties of the $25 \mathrm{~nm}$-thick and $96 \mathrm{~nm}$-wide Au NS array with periodicity of $300 \mathrm{~nm}$, similar to the one shown in Fig. 3(f), performed by evaporating macroscopic Au contact pads separated by $3 \mathrm{~mm}$ distance along the NS direction. A four probe measurement of the sheet resistance provides a value around $18 \Omega / \mathrm{sq}$ which is competitive with the best transparent conductive electrodes based on ITO as well as on metal networks $[41,51,52]$. In order to confirm these observations also on a local scale we measured the resistance of the same sample recurring to a conductive AFM equipped with a ResiScope module (Nano-observer, CSI) which simultaneously maps the topography and the current flowing from the biased sample to the virtually grounded conductive tip (diamond coated tip DD-ACTA-10). The strongly anisotropic current map reported in Fig. $3(\mathrm{~g})$ evidences that the high conductivity regions (reddish colours ranging up to $70 \mu \mathrm{A}$ ) are in registry with the topographic maxima and also demonstrates that each single NS is able to transport current over several millimetres. If we consider that the spectrally averaged optical transmittance of the $\mathrm{Au}$ NSs across the VIS-NIR spectrum (400-900 $\mathrm{nm}$ ) reads about $70 \%$ when using unpolarised light, we can conclude that our platform is also eligible for opto-electronic applications requiring flexible and transparent electrodes.

The optical extinction spectra with polarized light of Fig. 4(a) also show that the nanoelectrode array has a sharp LSP resonance whose spectral position can be easily and finely tailored by varying the height $h$ and width $w$ of the Au NSs (see the sketch depicted in inset of Fig. 4(a)). Here, the NSs width $w$ has been tuned from 96 to 93 and $70 \mathrm{~nm}$ by varying length $(L)$ of the overhanging structures at a constant thickness $h=25 \mathrm{~nm}$. We can also notice that the reduction of the width of the NSs results in a strong increase of direct optical transmittance due to progressive reduction of the effective metal coverage and at the same time incorporate a red-shift of the LSP resonance excited in TM polarization which respectively shifts from 582 to 590 and $607 \mathrm{~nm}$. Since the periodicity of the template and the thickness of Au NSs are kept constant, the optical response of the samples can be solely attributed to the width-induced changes of the NS aspect ratio $h / w$ which reads respectively $0.35,0.27$, and 0.26 . Alternatively, a similar fine tailoring of the LSP resonance can be obtained by increasing the height of the Au NSs at fixed width $w$. This possibility has

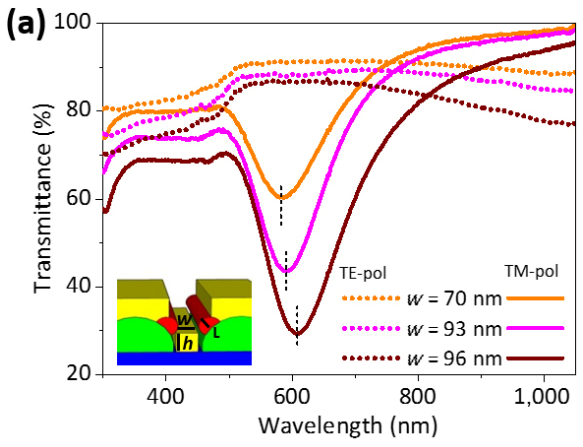

(c)

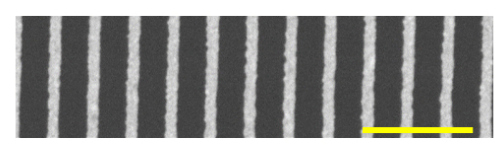

(d)

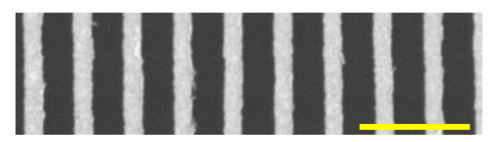

(e)
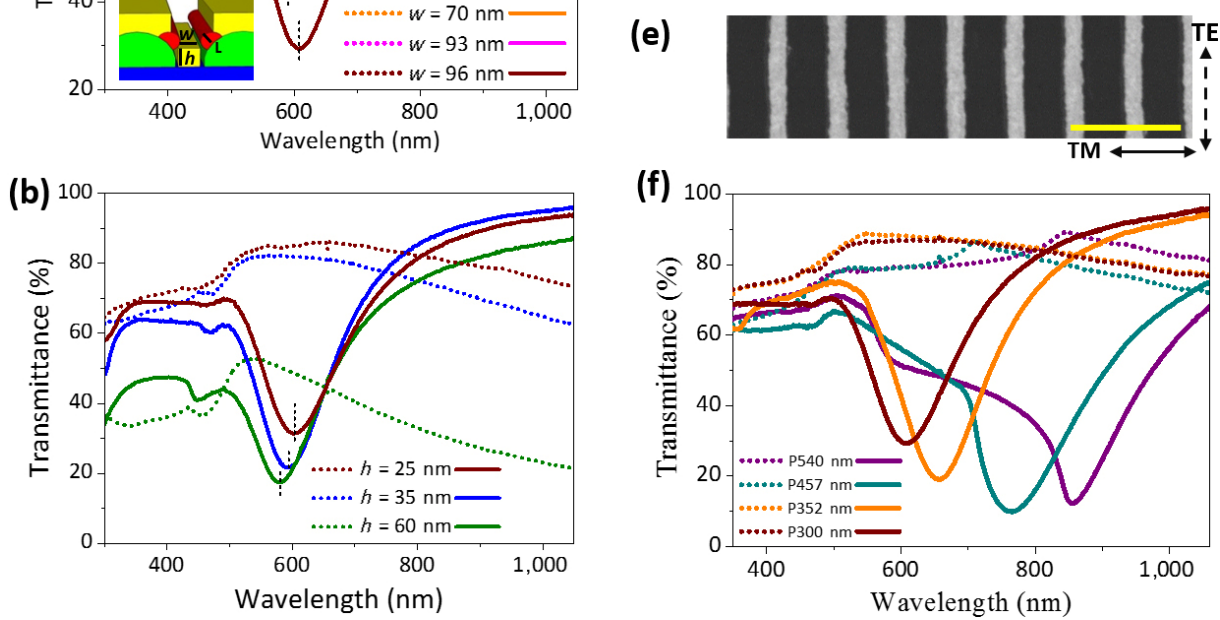

Figure 4 In (a), (b) and (f) optical direct transmission spectra through Au NSs gratings are measured with light parallel (TE: dotted lines) and perpendicular (TM: solid lines) with respect to NSs long axis, as depicted in the sketch at the right corner of panels (c)-(e). In (a) 25 nm-thick Au NSs gratings endowed with same periodicity of $300 \mathrm{~nm}$ but different lateral width of NSs (in the range from 96 to $70 \mathrm{~nm}$ ), in (b) the lateral width is fixed to $96 \mathrm{~nm}$ and the thickness is varied from 25 to $65 \mathrm{~nm}$ and in (f) transmittance spectra for plasmonic array of $25 \mathrm{~nm}$-thick NSs endowed with different periodicities 300 , $352,457$, and $540 \mathrm{~nm}$ are displayed. The SEM micrographs corresponding to NSs endowed with different widths (120, 197, and $200 \mathrm{~nm})$ and periodicities (reading respectively 352,457 , and $540 \mathrm{~nm}$ ) are represented in (c)-(e). The scale bars correspond to $1 \mu \mathrm{m}$. 
been explored by depositing $25 \mathrm{~nm}$-, $35 \mathrm{~nm}$ - and $65 \mathrm{~nm}$-thick $\mathrm{Au}$ NSs at normal incidence on a polymer stencil mask with fixed gap width $w=96 \mathrm{~nm}$. As shown in Fig. 4(b), the optical extinction measurements in TM polarization highlight a blueshift of the plasmonic resonance from $\lambda=607 \mathrm{~nm}$ to $\lambda=593 \mathrm{~nm}$ and $\lambda=579 \mathrm{~nm}$ as the thickness of Au NSs is respectively increased from 25 to 35 and $65 \mathrm{~nm}$ (aspect ratio $h / w$ reads $0.26,0.36,0.67)$.

We stress that in order to induce more substantial redshifts towards the relevant NIR range, one cannot further reduce the NS thickness $h$ since it is already close to the percolation limit of the Au film, in the range of $10 \mathrm{~nm}$ [9]. The percolation limit indicates a certain thickness of metallic film below which the film becomes granular and its metallic behaviour concerning conductivity gets disrupted. In the following we thus focus on the possibility of increasing the width $w$ of the NSs by enlarging the periodicity of the NSs array (from 300 to 352, 457 and $540 \mathrm{~nm}$ ) i.e., by changing the laser incident angle during LIL exposure (from $45^{\circ}, 35^{\circ}, 30^{\circ}$ and $25^{\circ}$ ). The determination of the morphological parameters of the NSs array was performed recurring to scanning electron microscopy (SEM) imaging of the samples: the aspect ratio $(h / w)$ of $\mathrm{Au}$ NSs is reduced as the lateral width $w$ of Au NSs is increased from 96 (Fig. 3(f)) to 120 (Fig. 4(c)), 197 ( Fig. 4(d)), and $200 \mathrm{~nm}$ (Fig. 4(e)) for constant height $h$ equal to $25 \mathrm{~nm}$. The optical response of such plasmonic gratings has been investigated by normal incidence far field spectroscopy in extinction configuration (see Fig. 4(f)). The TE polarization configuration (parallel to NSs) shows the typical behaviour of a thin Au film with a high transmittance in the range of $80 \%$ (dashed lines). When the grating periodicity $P$ increases to 352,457 , and $540 \mathrm{~nm}$, shallow transmittance peaks are visible in the TE spectrum at wavelengths around 545, 708, and $845 \mathrm{~nm}$ respectively in correspondence to the excitation of Rayleigh resonances (RR) of the Au NSs grating. RR characterizes the light scattering resonantly enhanced by each of the single nanostripes present in the grating $[53,54]$ as function of grating period at a fixed angle of incidence light. The wavelength $\lambda$ corresponding to $\mathrm{RR}$ can be determined following the relation $m \lambda=P\left(n_{\text {inc }} \sin \psi+n_{\text {diff }}\right)$ with $m$ (diffraction order) being equal to $1, \psi=0^{\circ}$ (light incidence angle) and an effective refractive index at the first and at the second interface $n_{\text {inc }}=1.0$ and $n_{\text {diff }}=1.58$ respectively [55]. In the case of the lowest periodicity $P=300 \mathrm{~nm}$ the RR peak at $475 \mathrm{~nm}$ is weakly observable since it overlaps with the strongly damped s-d interband transitions of Au. When the light electric field is oriented perpendicularly to NSs axis (TM polarization), LSP resonances take place evidencing the strong dichroism of all the samples. When the periodicity $P$ of the Au NSs is increased from 300 to 352 to 457 and $540 \mathrm{~nm}$, we observe a strong and remarkable redshift of the LSP mode which spans in the Vis-NIR range from 607 to $657,765 \mathrm{~nm}$ and up to $855 \mathrm{~nm}$ following the change in aspect ratio $h / w$ from 0.26 , to $0.21,0.13$ and 0.12 . We stress that the appearance of RR for larger pattern periodicities (from $P>350 \mathrm{~nm}$ ) is a further confirmation of the long range order of the Au NSs arrays which additionally show a broadly tunable localized plasmonic response on an ample range of wavelengths. It should be noted that as we observed RR for TE polarized illumination of our grating, analogous RR are indeed expected to manifest at about the same frequency for TM polarized light $[56,57]$. The presence of TM RR modes is not directly observable for the NS arrays of smaller periodicity as the associated weak transmission bumps are submerged in the broad and intense LSP optical background (wine and orange traces in Fig. 4(f)).
For larger grating periodicities, when the RR frequencies that we can monitor in the TE spectra overlap with the LSP modes (see in particular the purple trace-corresponding to $P=$ $540 \mathrm{~nm})$, we observe a surface lattice resonance $[58,59]$ i.e., a Fano-like coupling between the broad localised plasmonic resonances and the collective, sharp Rayleigh ones which gives rise to a significant asymmetric reshaping (sharpening) of the LSP peak of the individual nanostripe. In the extinction spectra shown in Fig. 4(f) we can also notice that, when the nanostripe width $w$ increases above $170 \mathrm{~nm}$ (purple traces), an additional extinction shoulder around $\lambda \approx 580 \mathrm{~nm}$ becomes observable. This shoulder, which is blue-shifted with respect to the dipolar LSP excitation, is assigned to the excitation of a multipolar plasmon mode of odd order $(n=3)$ while the even multipolar mode $(n=2)$ is not observed due to the light polarization here employed [21, 61-62].

Indeed, the plasmonic resonance shifted in the NIR can be exploited for an efficient management of low energy photons. Applications are possible in ultra-thin absorber (e.g., 2D semiconductors as $\mathrm{MoS}_{2}$ ) where the semi-transparent plasmonic electrode could convert NIR photons to hot electrons successively injected through a Schottky barrier directly in the conduction band of the semiconductor [25].

\subsection{Flat dimers and hybridization}

A key feature in view of the use of plasmonic nanostructures in nanospectroscopy applications is the possibility to exploit near field coupling and field enhancement when two resonant plasmonic structures are placed in close proximity. However, in the schemes presented so far (e.g., in Figs. 2 and 3), near field coupling among the plasmonic nanostructure cannot be achieved due to their large lateral separation exceeding $150 \mathrm{~nm}$. Nevertheless, the highly ordered templates as the one presented in Fig. 3(a), are ideal for the growth of a vertically stacked metal-insulator-metal (MIM) sequence where strong near field coupling, controlled by the insulator thickness, is expected. By properly engineering the shape of the Au NSs and the thickness of the dielectric gap it is possible to achieve hybridization of LSP dipolar resonances of individual nanostructures leading to an anti-bonding and bonding configurations: the former corresponds to the in-phase plasma oscillation of the two particles (electric dipole mode - ED) while the latter to the out-of-phase oscillation (magnetic dipole - MD) which are respectively weakly blue-shifted and strongly redshifted compared to the fundamental dipolar mode of the individual NS. We stress that numerical finite element simulations of local EM fields on similar dimeric structures predict a substantial field enhancement in correspondence to the MD resonance wavelength, as reported in the self-organised nano-dimers shown in Ref. [47].

Here, the synthesis of nano-dimers $\left(\mathrm{Au}-\mathrm{SiO}_{2}-\mathrm{Au}\right)$ is obtained by sequential deposition of $\mathrm{Au}, \mathrm{SiO}_{2}$ and $\mathrm{Au}$ on the polymer grating mask realized by LIL, following the same scheme presented in the sketch of Fig. 3(b). Au dimers are deposited on polymer templates at normal incidence with a nominal thickness of $25 \mathrm{~nm}$ via thermal evaporation after the evaporation of $2 \mathrm{~nm}$-thick Ti adhesion layer, while $\mathrm{SiO}_{2}$ of thickness $25 \mathrm{~nm}$ is deposited via RF magnetron sputtering. Once the growth of the dimer structure (all three layers of $\mathrm{Au}, \mathrm{SiO}_{2}$ and $\mathrm{Au}$ ) has been completed (each of the fabrication steps is represented schematically in Fig. 5(a)), the sample was immersed sequentially into acetone and isopropanol in an ultra-sonicating bath to lift-off the polymer lines. The lift-off took a bit longer time than the single metal layer. However, it was not difficult as the double-sided metallic overhanging structures, present on top 


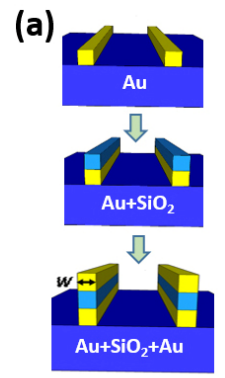

(d)
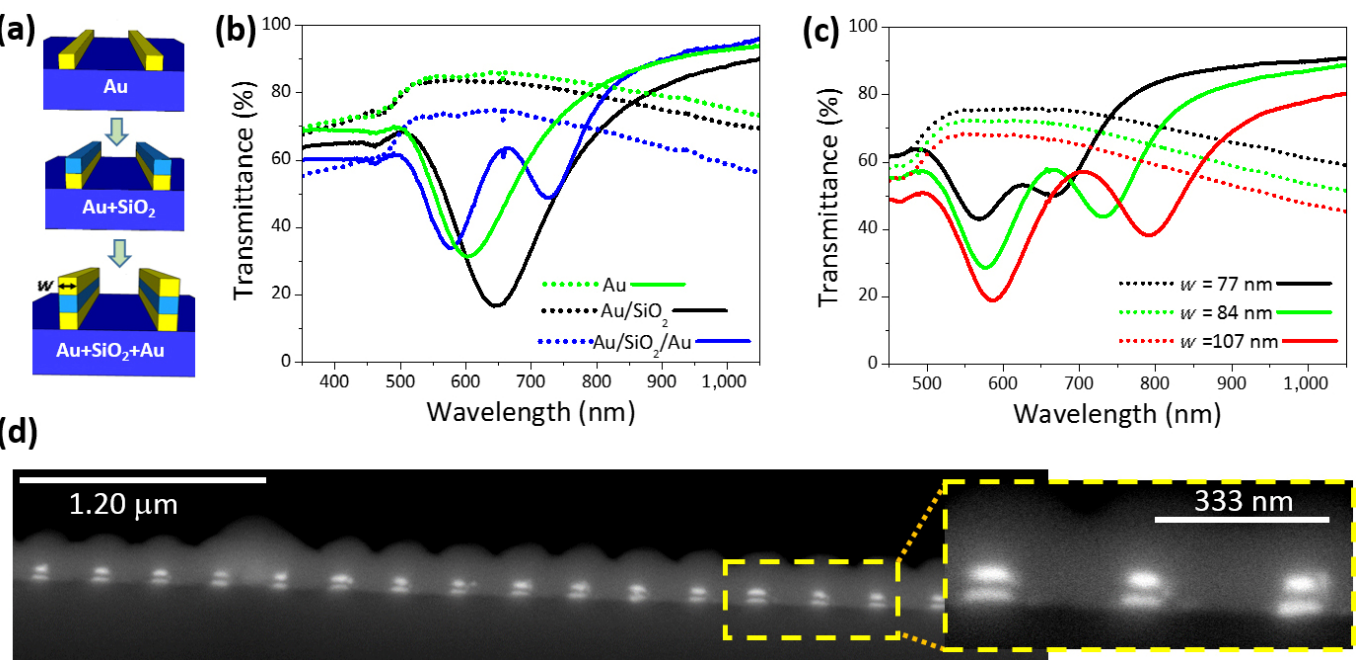

Figure 5 (a) Sequential steps of the nano-dimer formation process. (b) and (c) Optical transmission spectra acquired on arrays of nano-dimers $\left(\mathrm{Au} / \mathrm{SiO}_{2} / \mathrm{Au}\right.$ ) with light parallel (TE: dotted lines) and orthogonal (TM: solid lines) to their long axis at each process step (b): Au NSs (green) are capped with $\mathrm{SiO}_{2}$ stripes (black) and finally the double stack is covered with another Au NS (blue) to form a nano-dimer. (c) Nano-dimers array of same periodicity (300 nm) but with different lateral width $(w) 77,84$, and $107 \mathrm{~nm}$. (d) SEM micrograph acquired through backscattered electrons channel on the cross-section of the $\mathrm{Au} / \mathrm{SiO}_{2} / \mathrm{Au}$ sample. Scale bar is $1.2 \mu \mathrm{m}$ and $333 \mathrm{~nm}$ in the zoomed image.

of polymer resist ripples, prevent the capping of the polymer sidewalls from the normal incidence metal deposition and facilitate efficient lift-off. The cross-section of resulting structure is shown by SEM images in Fig. 5(d) where $\mathrm{Au} / \mathrm{SiO}_{2} / \mathrm{Au}$ stacks have been embedded into a thick sputter-deposited silica layer to avoid damaging the plasmonic structures during the cracking procedure. The double stack of Au NSs corresponds to the brighter regions in the SEM micrograph due to higher backscattering of electrons. The upper Au NSs are slightly narrower than the basal ones due to a shrinking of the opening gap of the mask during sequential Au deposition at normal incidence.

During each step of dimers nanofabrication process, we acquired direct optical transmission spectra on the NSs array and data are presented in Fig. 5(b) which highlights the response of $\mathrm{Au} \mathrm{NSs}$ (green trace), $\mathrm{Au} / \mathrm{SiO}_{2}$ stacks (black trace) and $\mathrm{Au} / \mathrm{SiO}_{2} / \mathrm{Au}$ dimers (blue trace). The addition of the $\mathrm{SiO}_{2}$ layer on the Au NSs does not alter the optical response in TE polarization. Conversely, when the system is probed in TM polarization the resonance is found red-shifted to $\lambda=647 \mathrm{~nm}$ (black traces), as expected when resonant plasmonic structures are embedded in a higher refractive index $\left(n_{\mathrm{Si}_{2}} \approx 1.75\right.$ estimated by optical measurements in reflection on a flat thin film). When the double stack $\mathrm{Au} / \mathrm{SiO}_{2}$ is further capped with the $\mathrm{Au}$ NS, we highlight the appearance of two spectrally separated minima in transmittance at lower and higher energies respectively. Their origin is attributed to plasmon hybridization in the triple stack $\left(\mathrm{Au} / \mathrm{SiO}_{2} / \mathrm{Au}\right)$ which enables the presence of well-defined electric dipolar resonance at $\lambda=575 \mathrm{~nm}$ and a magnetic dipolar mode at $\lambda=725 \mathrm{~nm}$. On the other side, if light is polarized parallel to the wires the spectrum shows the typical behaviour of a connected thin Au film with a monotonic reduction of transmittance moving towards the infrared spectrum. We stress that the MD mode is very sensitive to dispersion in the size of the dimers and to disorder in the pattern which in turn determine a strong inhomogeneous broadening and spectral shift, as was observed in the case of the self-organized nano-dimers of Ref. [47]. In present case, remarkably, we observe no broadening of the MD mode with respect to the ED mode which confirms the high degree of morphological order of the structures. Nevertheless, we exploit the sensitivity of the MD mode to the morphological parameters of the stacked $\mathrm{Au}$ and $\mathrm{SiO}_{2}$ nanostripes in order to efficiently tailor the plasmonic response of the dimeric metasurface in an ample range of wavelengths. According to hybridization theory, one way to control the optical response is to modulate the thickness of the dielectric spacer between the two resonators, as demonstrated in previous works [37, 47]. Indeed, by reducing the dielectric spacer thickness the NS near field coupling gets stronger, thus leading to a blue shift of ED mode and red-shift of MD mode. Vice-versa, by increasing the dielectric spacer thickness the opposite is true and two degenerate dipolar modes would be observed in the limit of an infinite dielectric slab instead of two detuned hybrid modes. In the present work we instead investigate the role of the lateral width $w$ (see sketch in Fig. 5(b)) of the dimers as a control parameter since it allows to control variations in the plasmonic behaviour of the monomeric metasurface (see Fig. 4).

We thus synthesized three different geometries where the lateral width of the dimers is varied from 77,84 to $107 \mathrm{~nm}$. The optical characterization of the arrays is reported in Fig. 5(c) which evidences that highly tunable hybridized plasmonic metasurfaces can be created on the $\mathrm{cm}^{2}$ area directly on glass or polymer templates. We observe a strong and monotonic red-shift of the MD mode from $\lambda=666 \mathrm{~nm}$ to $\lambda=731 \mathrm{~nm}$ and $\lambda=790 \mathrm{~nm}$ while the ED mode is modestly affected going from $\lambda=568 \mathrm{~nm}$ to $\lambda=576 \mathrm{~nm}$ and $\lambda=588 \mathrm{~nm}$, respectively. Scaling of $w$ is thus found more effective in tailoring the hybridized MD plasmonic resonance of the dimeric metasurfaces with respect to ED resonance of the monomeric counterpart. Furthermore, we highlight that strong field enhancement is expected in the proximity of dimers when the MD mode is resonantly excited: numerical simulations for $\mathrm{Au}$ NS dimers of similar geometry have shown an amplification factor $\left|E_{\text {local }} / E_{0}\right|$ above 10 , to be compared with a factor of 4 reported at the ED mode of monomers [47]. These features can make such dimeric metasurfaces an ideal platform in view of plasmon-enhanced sensing applications, where narrow line-shape and high tunability of the resonance are required to have the maximum near field amplification when the resonant nanostructures are optically tuned to the pump laser. As an example we mention the case of surface enhanced spectroscopies (like e.g., SERS) where the output signal is amplified following a $4^{\text {th }}$ power of the local electric field, and a shift of the pump laser towards 
the NIR spectral range allows to reduce the fluorescence background from substrate which hampers the detection of the weak SERS signal [44].

\section{Conclusions}

Transparent and conductive plasmonic metasurfaces are fabricated over large areas on transparent glass substrates as well as on flexible polymer foils demonstrating the ability to perform as multifunctional semi-transparent electrodes with sheet resistance in the range of $18 \Omega /$ sq. Nanofabrication of such metasurfaces is performed through an original low-cost variant of light interference lithography in Lloyd configuration which allows to fabricate ordered arrays of $\mathrm{Au}$ nanostripes (monomers) as well as of complex three-dimensional (3D)stacked $\mathrm{Au} / \mathrm{SiO}_{2} / \mathrm{Au}$ dimers covering uniform areas in the $\mathrm{cm}^{2}$ range. The plasmonic resonances of the nanostripes can be easily tailored to VIS-NIR spectral range by acting on their geometric aspect ratio. The possibility of vertically stack $\mathrm{Au} / \mathrm{SiO}_{2} / \mathrm{Au}$ dimers is exploited for achieving near-field coupling of individual nanostripes and for tailoring the hybridization of their localized plasmon resonances. In the bonding configuration (out-of-phase oscillation of the individual LSP-magnetic dipolar mode) one observes substantial redshifts of the resonances in the NIR range as well as substantial near-field enhancements [47], a feature of great interest in applications where low-energy photons management is mandatory like e.g., in plasmon enhanced spectroscopies. Due to the great lateral order of the plasmonic NS gratings, the multifunctional electrodes also exhibit coherent surface lattice resonances arising from the hybridization of Rayleigh anomalies with LSP modes of the individual nanostripes, thus providing an additional degree of freedom for tailoring the optical response of the template.

\section{Experimental details}

\subsection{Template fabrication}

In order to fabricate patterned template of photoresist by LIL method, the sample preparation was started with the cleaning procedure of the substrates through a series of ultra-sonic baths in isopropanol and acetone solvents respectively. At second step, a positive photoresist (AZ MIR 701) diluted with AZ-EBR solvent in a 1:2 mixture was spun on the substrate at $\sim 2 \mathrm{krpm}$ for $90 \mathrm{~s}$ in order to obtain a thin resist layer of $180 \mathrm{~nm}$. The thickness of the layer was measured by means of an AFM (Nanosurf Mobile S) and an optical profilometer (Smart WLI, GBS Ilmenau). After the spin coating, the sample was soft-baked at $100{ }^{\circ} \mathrm{C}$ for $300 \mathrm{~s}$ on a hotplate. At this point the sample was exposed under LIL set-up for $20 \mathrm{~s}$ with impinging power density $0.77 \mathrm{~mJ} /\left(\mathrm{cm}^{2} \cdot \mathrm{s}\right.$ ) (total incident energy density of $15.4 \mathrm{~mJ} / \mathrm{cm}^{2}$ on the substrates) which immediately follows a post-baking at $100{ }^{\circ} \mathrm{C}$ for $300 \mathrm{~s}$ on a hotplate. After that, the substrate was immersed in developer based on tetramethylammonium hydroxide (AZ $726 \mathrm{MIF}$ ) for $5 \mathrm{~s}$ and then rinsed with de-ionized water and blow-dried using $\mathrm{N}_{2}$ gas. Where the laser interference takes place on the sample, constructive interference fringes impress the positive tone resist that becomes locally etchable by the developer, and thus forms laterally disconnected polymer lines as the one sketched in Fig. 1(d). In case of thick $(\sim 1 \mu \mathrm{m})$ resist layer, by applying the mentioned recipe, one can still achieve the sinusoidal surface morphology but the valleys will not uncover the substrate surface.

\subsection{Morphological characterization}

The morphology of the patterned resist has been investigated by means of AFM operating in tapping mode (Ez Nanomagnetic, Nanosurf Mobile S and Nano-observer from CSI) equipped with high aspect ratio Si tips (ACLA-AppNano). We processed the topographic maps by using WSxM software [63] in order to evaluate the statistical parameters of the patterns (vertical amplitude, self-correlation functions, mean slope histograms and periodicity). Local electrical measurements have been acquired recurring to conductive AFM (Nano-observer from CSI) equipped with ResiScope module and diamond-coated tip (DD-ACTA-10).

\subsection{Building metal nanostripes}

Deposition of gold (Goodfellow, purity 99.99\%) on the patterned resist was accomplished in vacuum condition $\left(\sim 10^{-7} \mathrm{mbar}\right.$ range) at a rate of $6 \mathrm{~nm} / \mathrm{min}$ by means of a resistively heated alumina crucible. Deposition rate and thickness of metal have been evaluated through a calibrated quartz crystal microbalance. Lift-off of the resist nanowire mask has been completed by recurring to sonication in acetone bath for few minutes. The metal nanostructures have been investigated by means of a SEM (Hitachi SU3500) exploiting the backscattered electrons signal.

\subsection{Optical measurements}

In order to characterize the optical properties of the samples, we have carried out far-field spectroscopic transmittance measurements. The incident light source was a compensated deuterium-halogen lamp (DH-2000-BAL, Mikropak) emitting in the spectral range $300-2,500 \mathrm{~nm}$. Light was fiber coupled to a Glan-Thompson polarizer mounted on a rotating stage allowing variation of the incident light polarization state. Light transmitted by the samples has been coupled and analysed by means of a high-resolution solid-state spectrometer (HR4000, Ocean Optics).

\section{Acknowledgements}

Financial support is gratefully acknowledged from Ministero dell'Università e della Ricerca Scientifica (MIUR) through the PRIN 2015 Grant 2015WTW7J3, from Compagnia di San Paolo in the framework of Project ID ROL 9361, and from MAECI in the framework of the Italy-Egypt bilateral protocol. One of the authors (D.C.) acknowledges ICTP, Trieste, Italy for the TRIL Fellowship.

\section{List of symbols:}

$P$ : period of the pattern.

$\Lambda$ : laser wavelength.

$\theta$ : the angle between mirror and the laser axis.

$\phi$ : local slope of polymer lines.

$\alpha$ : deposition angle of gold.

$h$ : height of the nanostripes.

$w$ : width of the nanostripes.

$n$ : order of plasmon mode.

$m$ : diffraction order.

$n_{\text {inc }}$ : refractive index of medium through which light incident on diffractive medium.

$n_{\text {diff }}:$ effective refractive index of diffractive medium.

$\psi:$ incidence angle of light.

Funding note: Open Access funding provided by Università degli Studi di Genova within the CRUICARE Agreement.

Electronic Supplementary Material: Supplementary material 
(pattern periodicity tailoring, metal deposition scheme, large area order of pattern) is available in the online version of this article at https://doi.org/10.1007/s12274-020-3125-x.

Open Access This article is licensed under a Creative Commons Attribution 4.0 International License, which permits use, sharing, adaptation, distribution and reproduction in any medium or format, as long as you give appropriate credit to the original author(s) and the source, provide a link to the Creative Commons licence, and indicate if changes were made.

The images or other third party material in this article are included in the article's Creative Commons licence, unless indicated otherwise in a credit line to the material. If material is not included in the article's Creative Commons licence and your intended use is not permitted by statutory regulation or exceeds the permitted use, you will need to obtain permission directly from the copyright holder.

To view a copy of this licence, visit http://creativecommons.org/licenses/by/4.0/.

\section{References}

[1] Maier, S. A. Plasmonics: Fundamentals and Applications; Springer: New York, 2007.

[2] Yu, N. F.; Capasso, F. Flat optics with designer metasurfaces. Nat. Mater. 2014, 13, 139-150.

[3] Yu, H. K.; Peng, Y. S.; Yang, Y.; Li, Z. -Y. Plasmon-enhanced lightmatter interactions and applications. Npj Computat. Mater. 2019, 5,45 .

[4] Yu, T.; Wei, Q. S. Plasmonic molecular assays: Recent advances and applications for mobile health. Nano Res. 2018, 11, 5439-5473.

[5] Kristensen, A.; Yang, J. K. W.; Bozhevolnyi, S. I.; Link, S.; Nordlander, P.; Halas, N. J.; Mortensen, N. A. Plasmonic colour generation. Nat. Rev. Mat. 2017, 2, 16088.

[6] Atwater, H. A.; Polman, A. Plasmonics for improved photovoltaic devices. In Materials for Sustainable Energy; co-Published with Macmillan Publishers Ltd: UK, 2010; pp 1-11.

[7] Vala, M.; Homola, J. Flexible method based on four-beam interference lithography for fabrication of large areas of perfectly periodic plasmonic arrays. Opt. Express 2014, 22, 18778-18789.

[8] Van De Groep, J.; Spinelli, P.; Polman, A. Transparent conducting silver nanowire networks. Nano Lett. 2012, 12, 3138-3144.

[9] Giordano, M. C.; Repetto, D.; Mennucci, C.; Carrara, A.; De Mongeot, F. B. Template-assisted growth of transparent plasmonic nanowire electrodes. Nanotechnology 2016, 27, 495201.

[10] Xie, F.; Pang, J. S.; Centeno, A.; Ryan, M. P.; Riley, D. J.; Alford, N. M. Nanoscale control of Ag nanostructures for plasmonic fluorescence enhancement of near-infrared dyes. Nano Res. 2013, 6 , 496-510.

[11] Aksu, S.; Huang, M.; Artar, A.; Yanik, A. A.; Selvarasah, S.; Dokmeci, M. R.; Altug, H. Flexible plasmonics on unconventional and nonplanar substrates. Adv. Mat. 2011, 23, 4422-4430.

[12] Dunklin, J. R.; Forcherio, G. T.; Roper, D. K. Gold nanoparticlepolydimethylsiloxane films reflect light internally by optical diffraction and MIE scattering. Mater. Res. Express. 2015, 2, 085005.

[13] Laible, F.; Gollmer, D. A.; Dickreuter, S.; Kern, D. P.; Fleischer, M. Continuous reversible tuning of the gap size and plasmonic coupling of bow tie nanoantennas on flexible substrates. Nanoscale 2018, 10, 14915-14922.

[14] Prikhozhdenko, E. S.; Bratashov, D. N.; Gorin, D. A.; Yashchenok, A. M. Flexible surface-enhanced Raman scattering-active substrates based on nanofibrous membranes. Nano Res. 2018, 11, 4468-4488.

[15] Xu, K. C.; Zhou, R.; Takei, K.; Hong, M. H. Toward flexible surfaceenhanced Raman scattering (SERS) sensors for point-of-care diagnostics. Adv. Sci. 2019, 6, 1900925.

[16] Hasna, K.; Antony, A.; Puigdollers, J.; Kumar, K. R.; Jayaraj, M. K. Fabrication of cost-effective, highly reproducible large area arrays of nanotriangular pillars for surface enhanced Raman scattering substrates. Nano Res. 2016, 9, 3075-3083.

[17] Lee, K. L.; Chen, P. W.; Wu, S. H.; Huang, J. B.; Yang, S. Y.; Wei,
P. K. Enhancing surface plasmon detection using template-stripped gold nanoslit arrays on plastic films. ACS Nano 2012, 6, 2931-2939.

[18] Chen, C. H.; Yu, T. H.; Lee, Y. C. Direct metal transfer lithography for fabricating wire-grid polarizer on flexible plastic substrate. $J$. Microelectromechanical Syst. 2011, 20, 916-921.

[19] Meng, F. T.; Luo, G.; Maximov, I.; Montelius, L.; Chu, J. K.; Xu, H. Q. Fabrication and characterization of bilayer metal wire-grid polarizer using nanoimprint lithography on flexible plastic substrate. Microelectron. Eng. 2011, 88, 3108-3112.

[20] Aas, L. M. S.; Kildemo, M.; Martella, C.; Giordano, M. C.; Chiappe, D.; De Mongeot, F. B. Optical properties of biaxial nanopatterned gold plasmonic nanowired grid polarizer. Opt. Express 2013, 21, 30918-30931.

[21] Barelli, M.; Repetto, D.; De Mongeot, F. B. Infrared plasmonics via self-organized anisotropic wrinkling of Au/PDMS nanoarrays. ACS Appl. Polym. Mater. 2019, 1, 1334-1340.

[22] Song, M.; Park, J. H.; Kim, C. S.; Kim, D. H.; Kang, Y. C.; Jin, S. H.; Jin, W. Y.; Kang, J. W. Highly flexible and transparent conducting silver nanowire/ $\mathrm{ZnO}$ composite film for organic solar cells. Nano Res. 2014, 7, 1370-1379.

[23] Gong, S.; Zhao, Y. M.; Yap, L. W.; Shi, Q. Q.; Wang, Y.; Bay, J. A. P. B.; Lai, D. T. H.; Uddin, H.; Cheng, W. Fabrication of highly transparent and flexible NanoMesh electrode via self-assembly of ultrathin gold nanowires. Adv. Electron. Mater. 2016, 2, 1600121.

[24] Lyons, P. E.; De, S.; Elias, J.; Schamel, M.; Philippe, L.; Bellew, A. T.; Boland, J. J.; Coleman, J. N. High-performance transparent conductors from networks of gold nanowires. J. Phys. Chem. Lett. 2011, 2, 3058-3062.

[25] Knight, M. W.; Sobhani, H.; Nordlander, P.; Halas, N. J. Photodetection with active optical antennas. Science 2011, 332, 702-704.

[26] Mendes, M. J.; Luque, A.; Tobías, I.; Martí, A. Plasmonic light enhancement in the near-field of metallic nanospheroids for application in intermediate band solar cells. Appl. Phys. Lett. 2009, 95, 071105.

[27] Beck, F. J.; Polman, A.; Catchpole, K. R. Tunable light trapping for solar cells using localized surface plasmons. J. Appl. Phys. 2009, 105, 114310.

[28] Bouten, P. C. P.; Slikkerveer, P. J.; Leterrier, Y. Mechanics of ITO on plastic substrates for flexible displays. In Flexible Flat Panel Displays. Crawford, G. P. Ed.; John Wiley \& Sons, Ltd: Chichester, UK, 2005; pp 99-120.

[29] Wong, W. S.; Salleo, A. Flexible Electronics: Materials and Applications; Springer: New York, 2009.

[30] Gupta, R.; Rao, K. D. M.; Kiruthika, S.; Kulkarni, G. U. Visibly transparent heaters. ACS Appl. Mater. Interfaces 2016, 8, 1255912575 .

[31] Holze, R. P. Hendra, C. Jones, and G. Warnes: Fourier transform Raman spectroscopy-instrumentation and chemical applications, Ellis Horwood, Chichester, 1991, ISBN 0-13-327032-7, 311 pages, Preis: \$90.50. Ber. Bunsengesellschaft Phys. Chem. 1993, 97, 1500-1501.

[32] Zúñiga, W. C.; Jones, V.; Anderson, S. M.; Echevarria, A.; Miller, N. L.; Stashko, C.; Schmolze, D; Cha, P. D.; Kothari R.; Fong, Y. M. et al. Raman spectroscopy for rapid evaluation of surgical margins during breast cancer lumpectomy. Sci. Rep. 2019, 9, 14639.

[33] Noguez, C. Surface plasmons on metal nanoparticles: The influence of shape and physical environment. J. Phys. Chem. C 2007, 111, 3806-3819.

[34] Sekhon, J. S.; Verma, S. S. Rational selection of nanorod plasmons: Material, size, and shape dependence mechanism for optical sensors. Plasmonics 2012, 7, 453-459.

[35] Giordano, M. C.; Foti, A.; Messina, E.; Gucciardi, P. G.; Comoretto, D.; Buatier De Mongeot, F. B. SERS amplification from selforganized arrays of plasmonic nanocrescents. ACS Appl. Mater. Interfaces 2016, 8, 6629-6638.

[36] Sun, Y. H.; Jiang, L.; Zhong, L. B.; Jiang, Y. Y.; Chen, X. D. Towards active plasmonic response devices. Nano Res. 2015, 8, 406-417.

[37] Dmitriev, A.; Pakizeh, T.; Käll, M.; Sutherland, D. S. Gold-silica-gold nanosandwiches: Tunable bimodal plasmonic resonators. Small 2007, 3, 294-299.

[38] Fan, J. A.; Bao, K.; Sun, L.; Bao, J. M.; Manoharan, V. N.; Nordlander, P.; Capasso, F. Plasmonic mode engineering with templated selfassembled nanoclusters. Nano Lett. 2012, 12, 5318-5324.

[39] Theiss, J.; Aykol, M.; Pavaskar, P.; Cronin, S. B. Plasmonic mode 
mixing in nanoparticle dimers with nm-separations via substratemediated coupling. Nano Res. 2014, 7, 1344-1354.

[40] Baur, S.; Sanders, S.; Manjavacas, A. Hybridization of lattice resonances. ACS Nano 2018, 12, 1618-1629.

[41] Fortunato, E.; Ginley, D.; Hosono, H.; Paine, D. C. Transparent conducting oxides for photovoltaics. MRS Bull. 2007, 32, 242-247.

[42] Darvishzadeh-Varcheie, M.; Thrift, W. J.; Kamandi, M.; Ragan, R.; Capolino, F. Two-scale structure for giant field enhancement: Combination of Rayleigh anomaly and colloidal plasmonic resonance. Phys. Rev. Appl. 2019, 11, 054057.

[43] Mennucci, C.; Del Sorbo, S.; Pirrotta, S.; Galli, M.; Andreani, L. C.; Martella, C.; Giordano, M. C.; De Mongeot, F. B. Light scattering properties of self-organized nanostructured substrates for thin-film solar cells. Nanotechnology 2018, 29, 355301.

[44] Repetto, D.; Giordano, M. C.; Foti, A.; Gucciardi, P. G.; Mennucci, C.; De Mongeot, F. B. SERS amplification by ultra-dense plasmonic arrays on self-organized PDMS templates. Appl. Surf. Sci. 2018, 446, 83-91.

[45] Chowdhury, D.; Satpati, B.; Ghose, D. Temperature and high fluence induced ripple rotation on $\mathrm{Si}(100)$ surface. Mater. Res. Express 2016, 3, 125003 .

[46] Fucetola, C. P.; Korre, H.; Berggren, K. K. Low-cost interference lithography. J. Vac. Sci. Technol. B: Microelectron Nanometer Struct. 2009, 27, 2958.

[47] Giordano, M. C.; Longhi, S.; Barelli, M.; Mazzanti, A.; De Mongeot, F. B.; Valle, G. D. Plasmon hybridization engineering in self-organized anisotropic metasurfaces. Nano Res. 2018, 11, 3943-3956.

[48] Giordano, M. C.; De Mongeot, F. B. Anisotropic nanoscale wrinkling in solid-state substrates. Adv. Mater. 2018, 30, 1801840.

[49] Guerrisi, M.; Rosei, R.; Winsemius, P. Splitting of the interband absorption edge in Au. Phys. Rev. B 1975, 12, 557-563.

[50] Gong, S.; Schwalb, W.; Wang, Y. W.; Chen, Y.; Tang, Y.; Si, J.; Shirinzadeh, B.; Cheng, W. L. A wearable and highly sensitive pressure sensor with ultrathin gold nanowires. Nat. Commun. 2014, 5,3132

[51] Chen, H. Y.; Tu, M. C. Nanowire-based transparent conductive electrodes. In Nanowire Electronics. Shen, G. Z.; Chueh, Y. L., Eds.; Springer: Singapore, 2019; pp 159-200.
[52] Repetto, D.; Giordano, M. C.; Martella, C.; De Mongeot, F. B. Transparent aluminium nanowire electrodes with optical and electrical anisotropic response fabricated by defocused ion beam sputtering. Appl. Surf. Sci. 2015, 327, 444-452.

[53] Rayleigh, L. On the dynamical theory of gratings. Proc. R. Soc. Mathem. Phys. Charact. 1907, 79, 399-416.

[54] Rayleigh, L. III. Note on the remarkable case of diffraction spectra described by Prof. Wood. The Lond. Edinb. Dubl. Phil. l Mag. J. Sci. 1907, 14, 60-65.

[55] Park, J. M.; Nalwa, K. S.; Leung, W.; Constant, K.; Chaudhary, S.; Ho, K. M. Fabrication of metallic nanowires and nanoribbons using laser interference lithography and shadow lithography. Nanotechnology 2010, 21, 215301 .

[56] Hessel, A.; Oliner, A. A. A new theory of Wood's anomalies on optical gratings. Appl. Opt. 1965, 4, 1275-1279.

[57] Maradudin, A. A.; Simonsen, I.; Polanco, J.; Fitzgerald, R. M. Rayleigh and Wood anomalies in the diffraction of light from a perfectly conducting reflection grating. J. Opt. 2016, 18, 024004.

[58] Cherqui, C.; Bourgeois, M. R.; Wang, D. Q.; Schatz, G. C. Plasmonic surface lattice resonances: Theory and computation. Acc. Chem. Res. 2019, 52, 2548-2558.

[59] Kravets, V. G.; Kabashin, A. V.; Barnes, W. L.; Grigorenko, A. N. Plasmonic surface lattice resonances: A review of properties and applications. Chem. Rev. 2018, 118, 5912-5951.

[60] Laurent, G.; Félidj, N.; Aubard, J.; Lévi, G.; Krenn, J. R.; Hohenau, A.; Schider, G.; Leitner, A.; Aussenegg, F. R. Evidence of multipolar excitations in surface enhanced Raman scattering. Phys. Rev. B 2005, $71,045430$.

[61] Encina, E. R.; Coronado, E. A. Resonance conditions for multipole plasmon excitations in noble metal nanorods. J. Phys. Chem. C 2007, 111, 16796-16801.

[62] Payne, E. K.; Shuford, K. L.; Park, S.; Schatz, G. C.; Mirkin, C. A. Multipole plasmon resonances in gold nanorods. J. Phys. Chem. B 2006, 110, 2150-2154.

[63] Horcas, I.; Fernández, R.; Gómez-Rodríguez, J. M.; Colchero, J.; Gómez-Herrero, J.; Baro, A. M. WSXM: A software for scanning probe microscopy and a tool for nanotechnology. Rev. Sci. Instrum. 2007, 78, 013705 . 